\section{Isla de calor urbana superficial en el área metropolitana de Cuzco}

\author{
Julio Angeles ${ }^{1}\left(\mathbb{0}\right.$, José Flores ${ }^{2}{ }^{-}$, Roberto \\ Angeles $^{3} \odot$,Hugo Karam ${ }^{4} \odot$ \\ 1 Universidad Alas Peruanas; ${ }^{2}$ nstituto Geofisico del Perú, ${ }^{3}$ Universidad \\ Nacional del Centro del Perú; ${ }^{4}$ Universidad Federal do Rio de Janeiro \\ julio_as_1@hotmail.com; alicia.rocha.condor@hotmail.com; \\ roanvas@hotmail.com \\ DOI: 10.33412/pri.v12.1.2565
}

\section{()(1) $\$(0)$}

Resumen: La presente contribución estima la Intensidad de Isla de Calor Urbano Superficial (ICUSI durante el periodo 2001 - 2016 para el Área Metropolitana de Cuzco (AMC), localizado en Perú en el departamento de Cuzco. Para cuantificar la ICUS, se usó 2 métodos: el primero es el método de Streutker y el segundo método propuesto por José Flores (método de cuantil). Donde se utilizó data de sensoramiento remoto de Temperatura Superficial (TS) TS mensual y producto anual de tipo de cobertura de suelo (Land Cover Type) a $0.05^{\circ}$ de resolución, obtenido del sensor MODIS a bordo del satélite TERRA y AQUA. LOS resultados muestran que en el AMC, en los periodos diurnos, la ICUS máximo tiene el valor máximo en marzo $\left(4.6^{\circ} \mathrm{C}\right)$ y mínimo en julio $\left(0.5^{\circ} \mathrm{C}\right)$ y la ICUSIICUS nocturna presenta valor máximo en octubre $\left(2.6^{\circ} \mathrm{C}\right)$ y mínimo en marzo $\left(1.7^{\circ} \mathrm{C}\right)$ utilizando el método de cuantiles, ya que el área metropolitana de Cuzco no se ajusta a una superficie gaussiana.

Palabras clave: isla de calor; temperatura superficial; Cuzco.

Title: Surface Urban Heat Island in the metropolitan area of Cuzco

Abstract: This contribution estimates the Surface Urban Heat Island Intensity (IICUS) during the period 2001 - 2016 for the Metropolitan Area of Cuzco (AMC), located in Peru in the department of Cuzco. To quantify IICUS, 2 methods were used: the first is the Streutker method and the second method proposed by Jose Flores (quantile method). Where was used remote sensing data of monthly TS and annual product of land cover type (Land Cover Type) at 0.050 resolution, obtained from the MODIS sensor on board the TERRA and AQUA satellite. In the AMC, in the diurnal periods, the maximum IICUS shows the maximum values in March (4.6 C) and minimum values in July (0.5 C) and the nocturnal IICUS presents maximum values in October (2.6 C) and minimum values in March (1.7 C) using the quantile method, since the metropolitan area of Cuzco does not conform to a Gaussian surface.

Keywords: heat island; superficial temperature; Cuzco.

Tipo de artículo: Estudio.

Fecha de recepción: 29 de junio de 2020.

Fecha de aprobación: 5 de enero de 2021.

\section{Introducción}

Más de la mitad de la población mundial vive en áreas urbanas y se espera que el porcentaje continúe [1]. Las temperaturas elevadas en las áreas urbanas en comparación con las áreas rurales circundantes, conocidas como efecto de isla de calor urbano (ICU), han sido bien documentadas $[2,3,4,5]$ y está ocurriendo en ciudades de todo el mundo. Las razones por las cuales las áreas urbanas son más cálidas que las áreas rurales. Estos incluyen: se absorbe más radiación solar debido a que los edificios altos aumentan el área de superficie; una mayor cantidad de radiación solar se almacena como calor sensible debido a una mayor admisión térmica de superficies duras; menos radiación terrestre o de onda larga se pierde debido a un factor de visión del cielo restringido; hay menos evapotranspiración del suelo, plantas y cuerpos de aguas, ya que son reemplazadas por superficies duras, hay menos viento a nivel del suelo, lo que significa que el aire caliente no se disipa tan rápido, y; hay calor antropogénico que se consume de edificios, automóviles y otras fuentes [6].

El efecto de la ICU es ampliamente estudiado para examinar la contribución de la ciudad al aumento de la temperatura (por ejemplo, $[7,8,9,10,11,12,13,14,15]$. La ICU se define como cerca de la superficie diferencia de temperatura del aire entre áreas urbano y rural que lo rodea [16]. Se puede clasificar en tres tipos: canopy layer heat island (CLHI) (isla de calor en la capa del dosel) ii) boundary layer heat island (BLHI) (isla de calor en la capa limite) y iii) surface heat island (SHI) (Isla de calor superficial) [5].

La presente contribución propone la estimación de la ICUSIICUS a baja resolución durante el periodo (20012016) para el área metropolitana de Cuzco (AMC).

\section{Datos y métodos}

En el presente punto se muestra los subcapítulos, descripción del sitio, donde se muestra la geografía del lugar de estudio; en el subcapítulo instrumento, nos muestra el sensor utilizado el MODIS, para ello se consideró la obtención de uso de suelo y temperatura superficial, asimismo se presenta los subcapítulos estimación del ICUSI y método de cuantil, ambos subcapítulos indican la metodología utilizada para cuantificar la intensidad de isla de calor urbana superficial. 


\subsection{Descripción del sitio}

El Área Metropolitana de Cuzco (AMC) está localizado al sur-oeste de América del Sur, en Perú, departamento de Cuzco, extendiéndose aproximadamente entre $13.28 \mathrm{~S}$ a $13.8 \mathrm{~S}$ y $72.2 \mathrm{~W}$ a $71.7 \mathrm{~W}$. En la región Cusco, encontramos valores comprendidos entre 8 y $32^{\circ} \mathrm{C}$, los valores más altos se ubican en la selva norte y selva central, la cual incluye selva alta y baja. Los valores más bajos entre 8 y $12^{\circ} \mathrm{C}$ se ubican en las zonas de las cordilleras Vilcabamba (zona occidental del sector sierra central), Vilcanota (zona suroriental de la sierra central) y Paucartambo (divide la sierra central de la selva central). En Cusco sobre la selva norte y selva central oriental, los valores de temperatura mínima son mayores y oscilan entre 12 y $24^{\circ} \mathrm{C}$, teniendo una mayor distribución los valores entre 20 y $24^{\circ} \mathrm{C}$ en la selva norte. Los valles que atraviesan la selva norte son cálidos con valores entre 16 y $20^{\circ} \mathrm{C}$. En la sierra central oriental, los valores de temperatura mínima oscilan entre -8 y $12^{\circ} \mathrm{C}$, siendo los más bajos en la Cordillera Vilcanota con valores entre -8 y $-4^{\circ} \mathrm{C}$. En la sierra sur los valores fluctúan entre -8 y $4^{\circ} \mathrm{C}$, en dicha zona en las partes más altas llegan a alcanzar valores entre -8 y $-4^{\circ} \mathrm{C}$. En la sierra central occidental los valores fluctúan entre -4 y $12^{\circ} \mathrm{C}$. En el periodo multianual, en la sierra sur, la precipitación media es del orden de 500 a $1500 \mathrm{~mm}$, siendo menores a mayor altitud.

En tanto, en la selva norte, sobre la selva baja de la región Cusco, las precipitaciones fluctúan entre $2000-3000 \mathrm{~mm}$; mientras que en la selva alta entre 1000 a 2000 mm (Quillabamba y Machu Picchu). Sobre la selva central de Cusco, en lo que respecta a la selva alta, las precipitaciones climatológicamente presentan valores comprendidos entre 1500 y $5000 \mathrm{~mm}$. [17]. Las figuras $1 \mathrm{~b}$ y $1 \mathrm{~b}$ muestra la distribución horizontal del promedio mensual de la temperatura superficial (TS) del AMC para enero 2001 durante periodos del día y noche respectivamente. Para este mes, el máximo TS bajo el área urbana del AMC alrededor de $36 \mathrm{C}$ en el día y alrededor de $0{ }^{\circ} \mathrm{C}$ en periodos nocturnos.

En orden para distinguir el comportamiento diferente de TSTS a lo largo del año para áreas urbanas y rurales y su relación con la cobertura vegetal bajo el AMC, donde ha sido seleccionado 3 puntos con diferentes coberturas de superficie para el presente estudio. La Figura 1a muestra la localización rural (R) (72.05 S, 13.57 W), Urbano (U) (71.95 $\mathrm{S}, 13.53 \mathrm{~W})$ y frontera $(\mathrm{B})(72.01 \mathrm{~S}, 13.55 \mathrm{~W})$
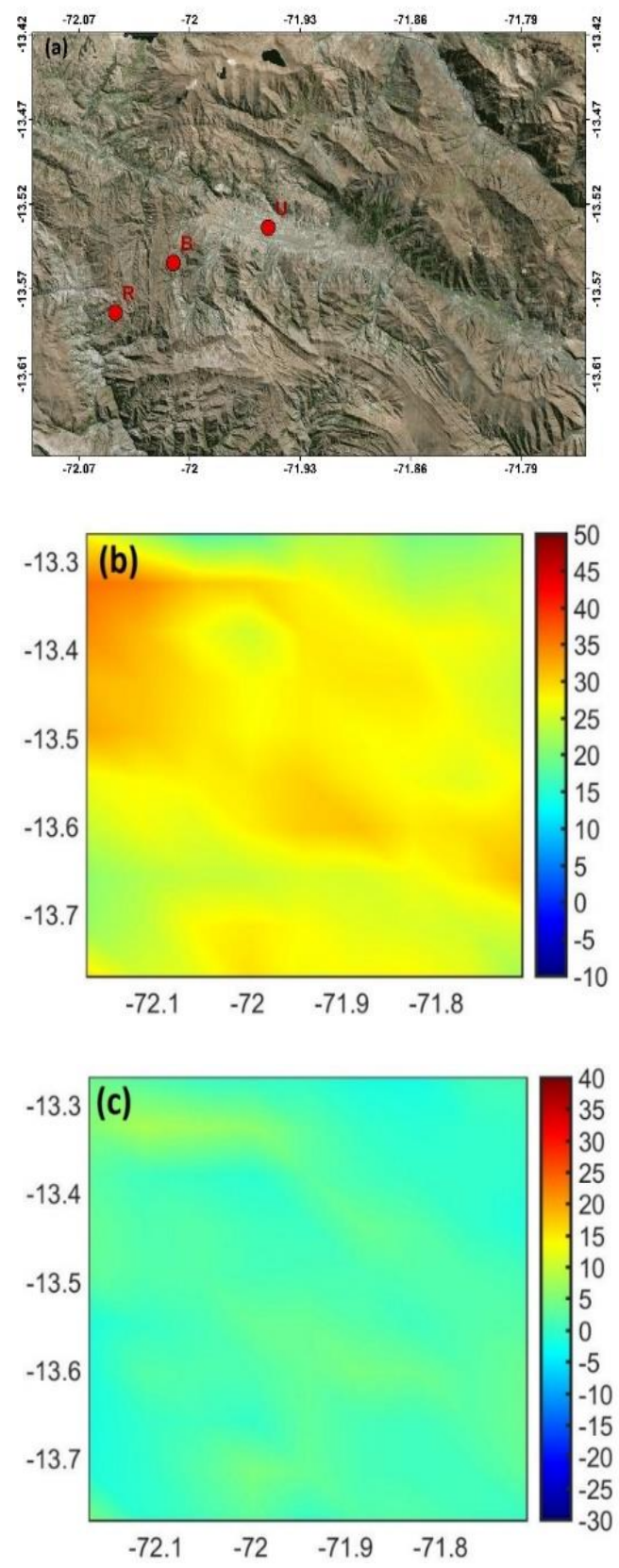

Figura 1 a) El dominio de la AMC y b) la media mensual de TS durante el día y c) periodos nocturnos en diciembre 2001. 


\subsection{Instrumento}

Se utilizó el sensor MODIS del Satélite AQUA y TERRA, donde se utilizó la TS [18].

En el presente estudio, se realizó un promedio mensual y escala de hasta una resolución de $5 \mathrm{Km}$, para analizar los efectos de reescalar en los patrones estadísticos de TS y luego compararlos con el producto MOD11C3 Global CMG, que es un promedio mensual compuesto, derivado del producto global diario MOD11C1. Estos datos se almacenan como valores de TS de cielo despejado durante un período de meses a una resolución de $0.05^{\circ}$ (5600 metros).

Con el objetivo de separar las áreas urbanas y rurales, se utilizó el producto MCD12C1 MODIS de cobertura terrestre tipo MCD12C1 con resolución $0.05^{\circ}$ para clasificar la superficie terrestre de acuerdo con el proyecto internacional Geosphere-Biosphere Project (IGBP), 17 tipos de cobertura del suelo a partir de las observaciones MODIS versión 4 siguiendo la contribución de [19].

\subsection{Estimación de la IICUSICUS}

Para determinar la Intesidad de la ICUIICUS se utilizó el Método de Streutker propuesta por Streutker [20].

La técnica usa un ajuste least-square fit de toda la isla de calor a una superficie gaussiana de la forma

$$
\begin{gathered}
T_{(x, y)}=T_{0}+a_{1} x+a_{2} y \\
+a_{0} e^{\left(-\frac{\left(x-x_{0}\right)^{2}}{2 a_{x}^{2}}-\frac{\left(y-y_{0}\right)^{2}}{2 a_{y}^{2}}\right)}
\end{gathered}
$$

Donde $T_{(x, y)}$ es la temperatura superficial total, incluidos los píxeles urbanos y rurales. $T_{0}, a_{1}$ y $a_{2}$ son los componentes constante y lineal de la temperatura rural, respectivamente. (Adaptado por[21].

\subsection{Método de cuantiles}

Este método propuesto por [21] para estimar la intensidad de la ICUS, donde se basa en el análisis estadístico de cuantiles de TS urbano y rural.

Asimismo, propuso la siguiente fórmula IICUS para una resolución de $5 \mathrm{~km}$ :

$$
I C U S=Q_{5}^{\text {urbano }}-Q_{3}^{\text {rural }}
$$

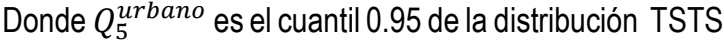
sobre el área urbana y $Q_{3}^{\text {rural }}$ es la mediana de la distribución TSTS sobre el área rural, ambas con una resolución de $5 \mathrm{~km}$,

\section{Resultados}

En el presente capítulo se muestra los resultados concernientes a los métodos utilizados: el método Streutker y cuantiles para obtener la intensidad de isla de calor urbano superficial diurno y nocturno, y su coeficiente de correlación entre ambos métodos utilizados.

\subsection{Variación temporal y espacial de la temperatura superficial en Perú}

La figura 2 muestra la distribución espacial del promedio mensual diurno de TS de Perú para enero y junio 2001 (estación de verano y otoño) obtenido del sensor MODIS
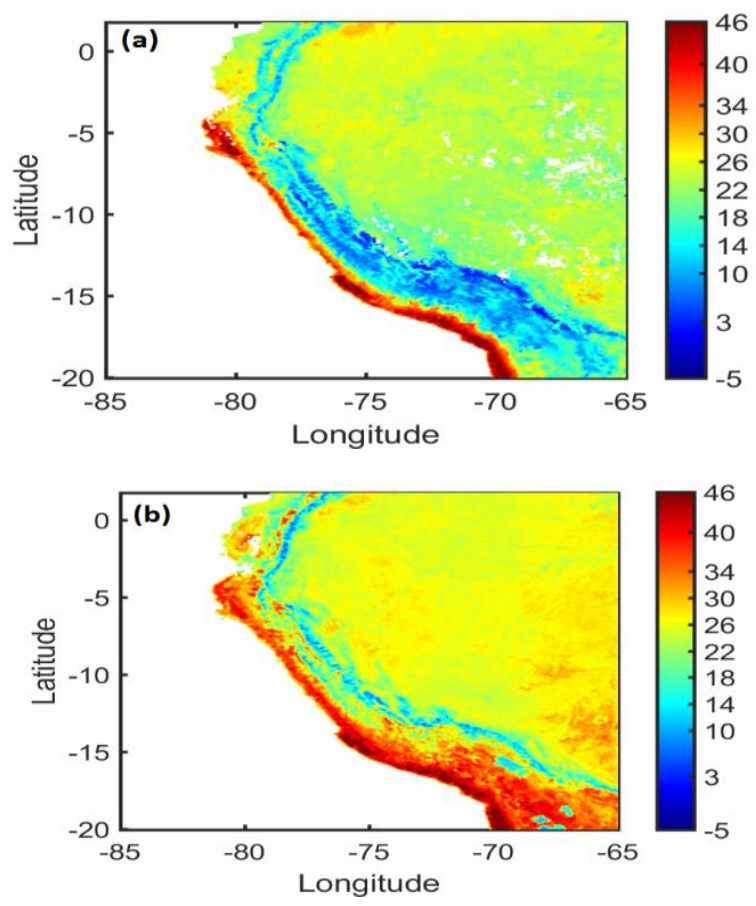

Figura 2. Distribución espacial del medio mensual diurno TS de Perú obtenido del producto MODIS TSS. Los datos han sido espaciados con una grilla de $0.05^{\circ} \times 0.05^{\circ}$

con el producto TS (MOD11C1) con $0.05^{\circ}$ resolución. Para este mes, la región de estudio, lquitos que esta entre $4 \mathrm{~S}$ a $2 \mathrm{~S}$ de latitud y $74 \mathrm{~W}$ a $73 \mathrm{~W}$, de longitud respectivamente.

\subsection{Isla de calor urbana superficial}

Los resultados para las magnitudes IICUS diurnas a largo plazo (2001-2016) y las extensiones espaciales para todos los meses sobre el AMC usando el método de Streutker, se presentan en la tabla 1. La intensidad IICUS mas alta para el periodo diurno ocurrió en diciembre (5.7 C) y se observó la intensidad IICUS más baja en julio (1.1 C). El área máxima de la huella se produjo en agosto $\left(305.9 \mathrm{~km}^{2}\right)$ y mínimo en febrero $\left(51.7 \mathrm{~km}^{2}\right)$. La tabla 1 también muestra el 
coeficiente de correlación (R-cuadrado) del ajuste de Gauss, con valores de 0.04 a 0.15 para todos los meses.

\subsection{Intensidad de la ICUSIICUS diurno y nocturno}

La figura 3a muestra el diagrama de dispersión entre ambos métodos por todos los meses en el periodo diurno.

Donde la pendiente de la línea recta que se ajusta a los datos es igual a 0.86 , la intersección es igual a 0.72 y el índice de correlación es igual a 0.71 . Este comportamiento se puede observar en la figura $8 a$, que muestra la comparación entre la evolución del tiempo de las intensidades de la ICUS con las desviaciones estándar utilizando Streutker y método cuantiles. Asimismo, en la figura $3 \mathrm{~b}$ para el periodo nocturno, la pendiente de la línea recta que se ajusta a los datos es igual a 0.89 , la intersección es igual a 0.57 y el índice de correlación es igual a 0.19 y en la figura $3 \mathrm{~b}$ se compara entre la evolución del tiempo de las intensidades del ICUS con las desviaciones estándar utilizando Streutker y método cuantiles.
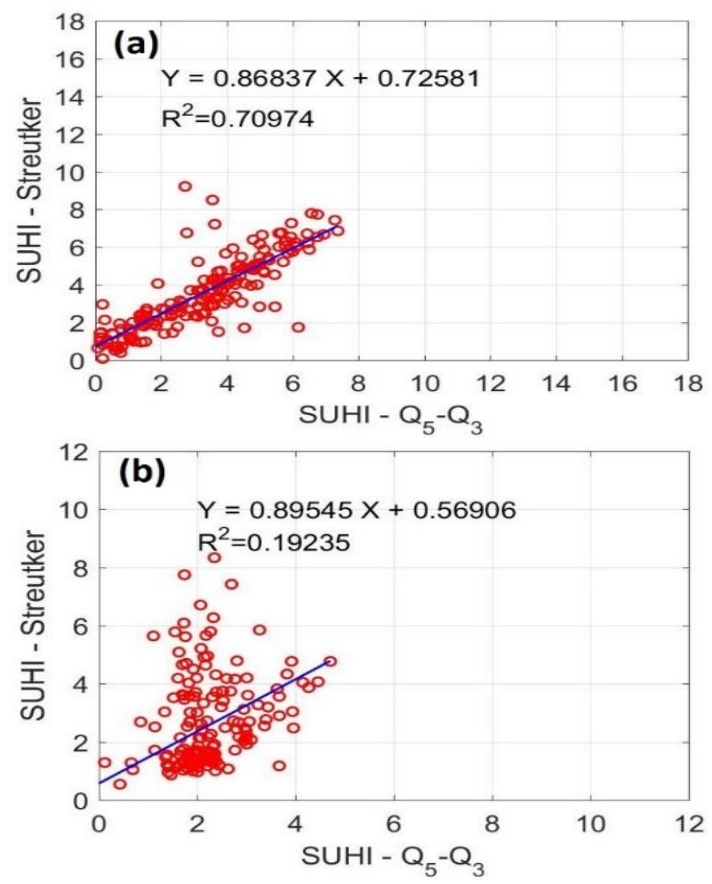

Figura 3 a) Scatterplot del IICUS en periodos diurno, obtenido con el método de Streutker's vs ICUSIICUS con la diferencia de cuantiles $Q_{5}^{\text {urban }}-Q_{3}^{\text {rural }}$ para la AMC, b) periodo nocturno.

Asimismo, en la figura 4 se observa que la intensidad de la ICUSI diurna y nocturna es mayor para el AMC en los meses de verano y primavera (setiembre-abril) y ligeramente inferior en los meses de mayo-agosto.
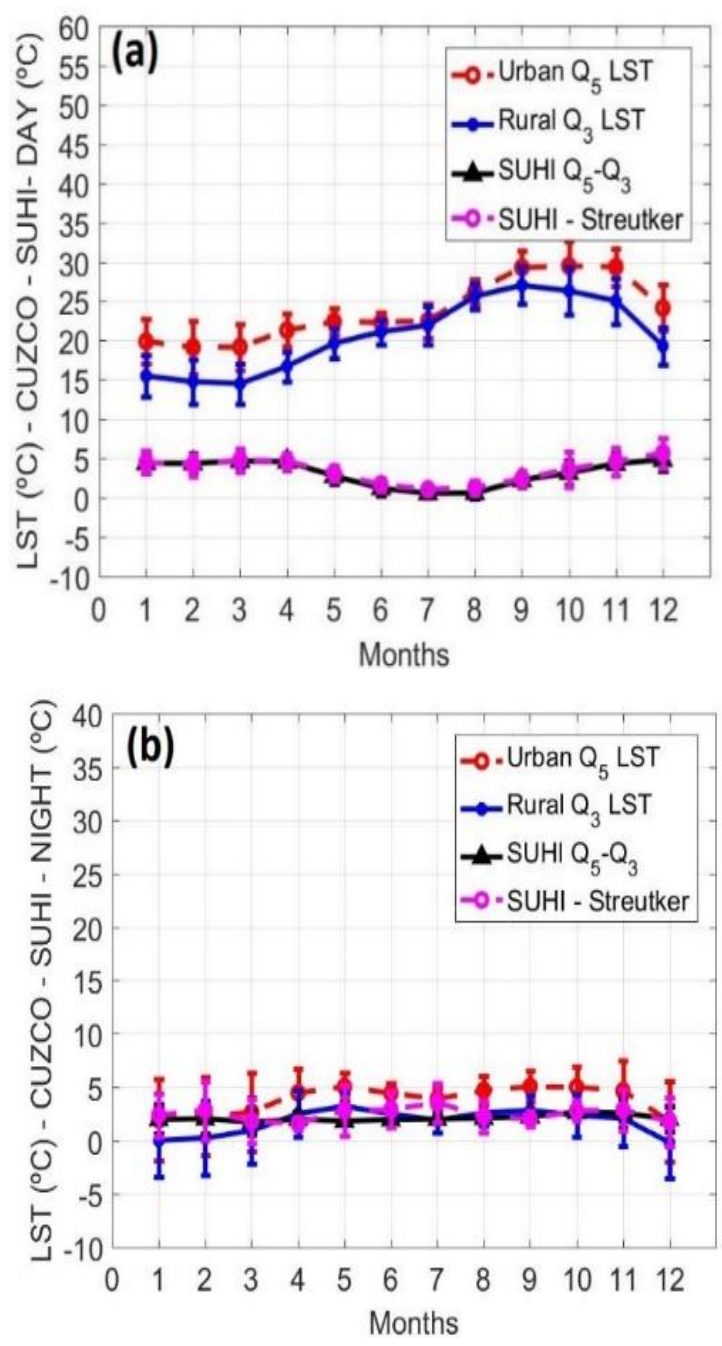

Figura 4 a) Evolución temporal de 2001-2016, media mensual diurno TS $\left({ }^{\circ} \mathrm{C}\right)$ con desviación estándar para periodo diurno de TS urbano $\left(Q_{5}\right)$ rural TS $\left(Q_{3}\right)$, intensidad del IICUS $\left({ }^{\circ} \mathrm{C}\right.$ ) para el $\left.\mathrm{AMC}, \mathrm{b}\right)$ periodo nocturno.

Para el AMC, los resultados que utilizan el método estadístico de cuantiles en periodos diurnos son que se muestra en la Tabla 2. El valor más alto del cuantil (Q5) para TS urbano se observó en octubre $\left(29.4^{\circ} \mathrm{C}\right)$ y el más bajo en febrero $\left(19.1^{\circ} \mathrm{C}\right)$. El valor máximo de TS rural medio (Q3) se observó en setiembre $\left(27^{\circ} \mathrm{C}\right)$ y mínimo en marzo $\left(14.5^{\circ} \mathrm{C}\right)$. Intensidades de IICUS diurnas obtenido con la ecuación 2 , muestra los valores máximos en marzo $\left(4.6^{\circ} \mathrm{C}\right.$ ) y valores mínimos en julio $\left(0.5^{\circ} \mathrm{C}\right)$. Comparando los resultados de las intensidades de ICUSIICUS usando ambos métodos (tablas 1 y 2), se observa un alto nivel de acuerdo entre ellos. 
Tabla 1 Media Diurna de la IICUS y extensión espacial con desviación estándar para el AMC usando el método desarrollado por Streutker (2002) del periodo 2001-2016.

\begin{tabular}{|c|c|c|c|c|c|c|}
\hline \multicolumn{7}{|c|}{ SUHI - Streutker ( $\left.{ }^{\circ} \mathrm{C}\right) \cdot \mathrm{MAC}$} \\
\hline Month & $\mathrm{T}_{0}\left({ }^{\circ} \mathrm{C}\right)$ - Rural & $A_{0}\left({ }^{0} \mathrm{C}\right)$ & $\mathrm{a}_{t}^{1}(\mathrm{~km})$ & $a_{y}^{1}(k m)$ & Footprint area $\left(\mathrm{km}^{2}\right)^{2}$ & RSquare \\
\hline $\begin{array}{l}\text { Jantury } \\
\end{array}$ & $15.7 \pm 2.5$ & $4.9 \pm 1.4$ & $111.0 \pm 5.7$ & $4.8 \pm 2.5$ & $54.0 \pm 38.8$ & 0.10 \\
\hline February & $14.8 \pm 2.8$ & 4.211.4 & $9.7 \pm 3.3$ & $5.7 \pm 2.7$ & $51.7 \pm 30,3$ & 0.11 \\
\hline March & $14.6 \pm 2.5$ & 4.7t1.4 & $8.4 \pm 0.9$ & $7.7 \pm 4.2$ & $65.0 \pm 37.1$ & 0.15 \\
\hline April & $16.8 \pm 1.8$ & 4.6t1.1 & $12.9 \pm 19.9$ & $6.9 \pm 1.8$ & $91.7 \pm 144.8$ & 0.11 \\
\hline May & $19.7 \pm 1.8$ & $3.1 \pm 0.9$ & $6.1 \pm 3.9$ & $7.3 \pm 4.2$ & $39.5 \pm 23.9$ & 0.05 \\
\hline June & $20.9+1.4$ & $1.7 \pm 0.8$ & 2.4559 .8 & $11.2+6.3$ & $221.3+149.1$ & 0.04 \\
\hline July & $21.7+2.3$ & $1.1+0.6$ & 8.7181 .9 & $5.3+22.9$ & $120.4 \pm 561.3$ & 0.05 \\
\hline August & 25.41.1.5 & $1.3+0.9$ & $29.34+5.4$ & $6.8+8.4$ & $305.9+445.9$ & 0.04 \\
\hline September & $27.0+2.2$ & $2.4+00.9$ & $16.7 \pm 27.7$ & 10.144 .8 & $152.9 \pm 236.9$ & 0.05 \\
\hline October & $26.3 \pm 2.9$ & $3.6 \pm 2.2$ & $10.2+20.2$ & $6.8 \pm 4.9$ & $75.6 \pm 186.4$ & 0.05 \\
\hline November & $25.1+2.7$ & $4.6 \pm 1.7$ & $9.3 \pm 11.6$ & $7.2 \pm 3.3$ & $75.6 \pm 1201.3$ & 0.08 \\
\hline December & $19.5+2.4$ & $5.7 \pm 1.8$ & $19.5 \pm 10.4$ & $5.7 \pm 4.3$ & $115.1 \pm 254.2$ & 0.10 \\
\hline
\end{tabular}

Tabla 2 Promedio mensual diurno de TSS $\left({ }^{\circ} \mathrm{C}\right)$ con su desviación estándar para áreas rurales y urbanas y la intensidad de la ICUSIICUS $\left({ }^{\circ} \mathrm{C}\right)$.

\begin{tabular}{|c|c|c|c|c|c|}
\hline \multirow[b]{2}{*}{ Month } & \multicolumn{2}{|c|}{ Urban LST $\left({ }^{\circ} \mathrm{C}\right) \cdot$ MA } & \multicolumn{2}{|c|}{ Rural LST $\left({ }^{\circ} \mathrm{C}\right) \cdot \mathrm{MAC}$} & \multirow{2}{*}{ 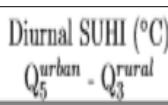 } \\
\hline & $Q_{3}$ & $0_{5}$ & $Q_{3}$ & $Q_{5}$ & \\
\hline January & $19.4 \pm 2.8$ & $19.8 \pm 2.8$ & $15.5 \pm 2.6$ & $19.8 \pm 2.3$ & $4.4 \pm 1.3$ \\
\hline February & $18.5+3.3$ & $19.1 \pm 3.4$ & $14.7 \pm 2.8$ & $18.6 \pm 2.8$ & $4.4 \pm 1.4$ \\
\hline March & $18.7 \pm 2.8$ & $19.2+2.9$ & $14.5+2.6$ & $18.5+2.5$ & 4.6t1.1 \\
\hline April & $20.8 \pm 2.0$ & $21.3 \pm 2.1$ & $16.7 \pm 1.9$ & $20.7 \pm 1.7$ & 4.6t1.1 \\
\hline May & $22.1+1.7$ & $22.4+1.7$ & $19.6 \pm 1.9$ & 23.1.1. 6 & $2.8 \pm 1.0$ \\
\hline June & $22.0 \pm 1.3$ & $22.3+1.2$ & $21.1 \pm 1.5$ & $23.8+1.2$ & $1.2 \pm 0.8$ \\
\hline July & $22.4 \pm 2.2$ & $22.5 \pm 2.1$ & $21.9 \pm 2.4$ & $24.7 \pm 2.2$ & $0.5 \pm 0.5$ \\
\hline August & $25.9+1.5$ & $26.2 \pm 1.5$ & $25.6 \pm 1.6$ & $28.4 \pm 1.5$ & $0.6 \pm 0.6$ \\
\hline September & $28.7 \pm 2.1$ & $29.2 \pm 2.2$ & $27.0 \pm 2.3$ & $30.9 \pm 1.8$ & $2.2 \pm 0.8$ \\
\hline October & $28.8 \pm 3.1$ & $29.4 \pm 3.2$ & $26.3 \pm 3.1$ & $30.9 \pm 2.7$ & $3.2 \pm 1.5$ \\
\hline November & $28.3 \pm 2.2$ & $29.4 \pm 2.4$ & $25.0 \pm 2.9$ & $29.4 \pm 2.6$ & $4.3 \pm 1.5$ \\
\hline December & $23.4 \pm 2.7$ & $24.1 \pm 2.9$ & $19.3 \pm 2.4$ & $23.9 \pm 2.4$ & $4.8 \pm 1.5$ \\
\hline
\end{tabular}

Durante el periodo nocturno, la TSS rural media cae significativamente para el cuantil 0.95 (Q5) del área urbana y la mediana (Q3) del área rural para el AMC. Para el AMC, los resultados usando el método estadístico de cuantiles en los periodos nocturnos se muestra en la tabla 3 . El valor más alto del cuantil (Q5) para TS urbano se observó en setiembre $\left(5.1^{\circ} \mathrm{C}\right)$ y el más bajo en diciembre $\left(1.8^{\circ} \mathrm{C}\right)$. El valor máximo de mediana TS rural (Q3), se observó en mayo $\left(3.2{ }^{\circ} \mathrm{C}\right)$ y el mínimo en diciembre $\left(-0.2{ }^{\circ} \mathrm{C}\right)$. Intensidades IICUS nocturnas obtenidas con el Ecuación 2, presento valor máximo en octubre $\left(2.6^{\circ} \mathrm{C}\right)$ y mínimo en marzo $\left(1.7^{\circ} \mathrm{C}\right)$.
Tabla 3 Promedio mensual nocturno de TS (C) con su desviación estándar para áreas rurales y urbanas y la intensidad del IICUS (C)

\begin{tabular}{|c|c|c|c|c|c|}
\hline \multirow[b]{2}{*}{ Month } & \multicolumn{2}{|c|}{ Urban LST $\left({ }^{\circ} \mathrm{C}\right)$ - MA } & \multicolumn{2}{|c|}{ Rural LST $\left({ }^{\circ} \mathrm{C}\right)$ - MAC } & \multirow{2}{*}{$\begin{array}{c}\text { Nocturnal SUHI I }\left({ }^{\circ} \mathrm{C}\right. \\
Q_{5}^{\text {surben }}-Q_{2}^{\text {rural }}\end{array}$} \\
\hline & $Q_{3}$ & $0_{5}$ & $0_{3}$ & $Q_{5}$ & \\
\hline January & $1.3 \pm 4.0$ & $1.9 \pm 3.8$ & $0.0 \pm 3.4$ & $3.1 \pm 3.3$ & $1.9 \pm 1.3$ \\
\hline February & $1.9 \pm 3.7$ & $2.3 \pm 3.7$ & $0.2 \pm 3.4$ & $3.6 \pm 3.1$ & $2.0 \pm 1.4$ \\
\hline March & $2.2 \pm 3.8$ & $2.6 \pm 3.7$ & $0.9 \pm 3.1$ & $4.2 \pm 2.8$ & $1.7 \pm 1.2$ \\
\hline April & $4.2 \pm 2.3$ & $4.5 \pm 2.3$ & $2.5 \pm 2.2$ & $5.6 \pm 2.1$ & $1.9 \pm 0.6$ \\
\hline May & $4.8 \pm 1.3$ & $5.0 \pm 1.3$ & $3.2 \pm 1.2$ & $6.3 \pm 1.1$ & $1.8 \pm 0.3$ \\
\hline June & $4.2 \pm 0.9$ & $4.4 \pm 0.9$ & $2.5 \pm 0.9$ & $5.8 \pm 0.8$ & $1.9 \pm 0.3$ \\
\hline July & $3.7 \pm 1.1$ & $3.9 \pm 1.0$ & $1.9 \pm 1.1$ & $5.2 \pm 1.1$ & $2.0 \pm 0.4$ \\
\hline August & $4.6 \pm 1.3$ & $4.7 \pm 1.3$ & $2.6 \pm 1.2$ & $6.3 \pm 0.9$ & $2.1 \pm 0.2$ \\
\hline September & $4.8 \pm 1.5$ & $5.1 \pm 1.5$ & $2.8 \pm 1.4$ & $6.2 \pm 1.4$ & $2.2 \pm 0.5$ \\
\hline October & 4.6 $6 \pm 1.9$ & $5.0+1.9$ & $2.4 \pm 1.9$ & $5.7 \pm 1.7$ & $2.6 \pm 0.6$ \\
\hline November & $4.2+2.9$ & $4.6 \pm 2.8$ & $2.1+2.6$ & $5.6 \pm 2.2$ & $2.6 \pm 1.3$ \\
\hline December & $1.2+3.8$ & $1.8 \pm 3.8$ & $-0.2 \pm 3.3$ & $2.7 \pm 3.3$ & $2.0+1.2$ \\
\hline
\end{tabular}

\section{Conclusión}

El objetivo principal de la presente contribución es estimar el IICUS durante el periodo (2001-2016) a una resolución $\left(0.05^{\circ}\right)$ para el AMC, utilizando datos del sensor MODIS, con el método de Streutker y análisis estadístico de cuantiles basado en diferencias de cuantiles de TSTS sobre las áreas urbano y rural separadas con el producto Land Cover Type MODIS. De acuerdo con el análisis y las pruebas presentadas en este documento, se concluyó.

Para el AMC, los resultados de ambos métodos muestran un nivel aceptable. Intensidades de ICUS diurnas muestra los valores máximos en marzo $\left(4.6^{\circ} \mathrm{C}\right)$ y valores mínimos en julio $\left(0.5^{\circ} \mathrm{C}\right)$ y la IICUS nocturnas presenta valores máximos en octubre $\left(2.6^{\circ} \mathrm{C}\right)$ y valores mínimos en marzo $\left(1.7^{\circ} \mathrm{C}\right)$

La correlación entre los métodos de cuantiles y de Streutker en el periodo diurno, se obtuvo que la pendiente de la línea recta que se ajusta a los datos es igual a 0.86 , la intersección es igual a 0.72 y el índice de correlación es igual a 0.71 . Y para el periodo nocturno, la pendiente de la línea recta que se ajusta a los datos es igual a 0.89 , la intersección es igual a 0.57 y el índice de correlación es igual a 0.19 Asimismo, el método de cuantiles es que se puede usar como método alternativo cuando la forma de la ciudad no es elipsoidal o la resolución espacial es tan alta que no permite un ajuste de la superficie gaussiana.

\section{Referencias}

[1] World Health Organization. Urban Population Growth. Retrieved January20, 2016, 2014. from http://www.who.int/gho/urban health/situation trends/urbanpopulation growth text/en/.

[2] M. J. Alcoforado, \& A: Matzarakis. Planning urban climates In different climatic zones. Geographicalia, 57, 5-39, 2010.

[3] A. Dimoudi, A: Kantzioura, S. Zoras, C. Pallas \& P. Kosmopoulos. Investigation of urban microclimate parameters in an urban center. Energy \&Buildings, 64, 1-9, 2013. 
[4] M. J. Maloley. Thermal remote sensing ofurban heat island effects: GreaterToronto area. Geological survey of Canada open file 283, 2010.

[5] J. Voogt \& T. Oke. Thermal remote sensing of urban climates. RemoteSensing of Environment, 86(3), 370-384, 2003

[6] T. Oke. Boundary Layer Climates, 2nd Edition. Taylor and Francis Group, 1987.

[7] A. J. Arnfield : Two decades of urban climate research: a review Of turbulence, exchanges of energy and water, and the urban heat island. Int. J. Climatol. 23, 1-26, , 2003.

[8] W. T.Chow \& M. Roth. Temporal dynamics of the urban heat island of Singapore. International Journal of Climatology, 26(15), 2243- 2260, 2006.

[9] I. Eliasson. Urban nocturnal temperatures, street geometry and land use. Atmospheric Environment, 30(3), 379-392, 1996.

[10] Y. H. Kim \& J. J. Baik. Spatial and temporal structure of the urban heat island in Seoul. Journal of Applied Meteorology and Climatology, 44(5), 591, 2005

[11] K. Klysik \& K. Fortuniak.. Temporal and spatial characteristics of the urban heat island of Lodz, Poland. Atmospheric Environment, 33, 3885-3895, 1999

[12] H. E. Landsberg. The urban climate, Academic Press.Vol. 28, 1981.

[13] T. R. Oke \& G. B. Maxwell. Urban heat island dynamics in Montreal and Vancouver. Atmospheric Environment (1967), 9(2),
$191-200,1975$

[14] M. Roth. Review of urban climate research in (sub)tropical Regions. International. Journal of Climatology, 27, 1859-1873, 2007.

[15] M. Santamouris \& D. Kolokotsa. Urban climate mitigation techniques. Routledge, 2016.

[16] T. R. Oke. The energetic basis of the urban heat island Quarterly Journal of the Royal Meteorological Society, 108(455), 1-24, 1982.

[17] Servicio Nacional de Meteorología e Hidrología del Perú, 2018, Caracterización Climática de las regiones Apurimac y Cusco

[18] B. Dousset. \& F. Gourmelon. Satellite multi-sensor data analysis of urban surface temperatures and land cover. J. Photogramm Remote Sens. 58, 43(54), 2003.

[19] Schneider. Mapping urban areas by fusing multiple sources of coarse resolution remotely sensed data. Photogramm. Eng.Remote Sens. 69, 1377(1386), 2002.

[20] D. Streutker. Satellite-measured growth of the urban heat island of houston, texas. International Journal of Remote Sensing 23, 25952608,2002

[21] J. Flores, A. Pereira \& H. Karam. Estimation of long term low Resolution surface urban heat intensities for tropical cities using modis remoste sensing data. Urban Climate 17, 32(66), 2016. 